\title{
Evidence for Stimulated Emission in Silicon Nanocrystal Microspheres
}

\author{
H. Chen ${ }^{*}$, J.-Y. Sung ${ }^{\ddagger}$, A. Tewary ${ }^{* *}$, M. Brongersma ${ }^{* *}$, J. H. Shin ${ }^{\ddagger}$, and P.M. Fauchet ${ }^{\dagger}$ \\ * Department of Physics \& Astronomy, ${ }^{\dagger}$ Department of Electrical and Computer Engineering, \\ University of Rochester, Rochester NY, 14627, USA \\ $\$$ Department of Physics, Korea Advanced Institute of Science and Technology, Taejon, Korea \\ ** Geballe Laboratory for Advanced Materials, Stanford University, Stanford CA, 94305, USA
}

\begin{abstract}
Silicon has been used in all photonic functions except lasing. We report evidence for stimulated emission in silicon nanocrystal microspheres under strong pulsed excitation.
\end{abstract}

\section{INTRODUCTION}

On-chip optical interconnects are one of the most promising solutions to the "interconnect bottleneck" identified by the International Technology Roadmap of Semiconductors [1]. While many building blocks for onchip optical interconnects including silicon-based waveguides, modulators and detectors [2, 3, 4] have achieved significant progress over the past few years, an electrically injected silicon laser is still missing. Compared to external light sources, an on-chip electrically injected silicon laser can greatly decrease the cost and complexity thereby making on-chip optical interconnects practical.

As an indirect bandgap semiconductor, bulk silicon has low light emission efficiency. However, this can be modified by using low dimensional silicon. In the past few years, stimulated emission and light amplification have been demonstrated in nanocrystalline silicon (nc-Si) systems prepared by ion implantation [5], plasma enhanced chemical vapor deposition (PECVD) [6] and RF magnetron sputtering [7]. All these experiments were done using a planar film with single pass amplification. However, to achieve lasing, a resonant cavity is needed. Recently, microspheres have attracted attention in the area of cavity enhanced non-linear optical effects due to their high quality factor [8, 9]. In this work, silicon nanocrystals (Si-nc) coated high-Q silica microspheres are investigated to explore the possibility of lasing.

\section{EXPERIMENTAL CONDITIONS}

The high-Q silica microspheres are fabricated at Stanford University. $\mathrm{A} \mathrm{CO}_{2}$ laser beam is focused on the tip of a piece of silica fiber. The tip is melted and reshapes itself into a sphere of about $100 \mu \mathrm{m}$ diameter via surface tension. A layer of amorphous $\mathrm{SiO}_{\mathrm{x}}$ is then deposited onto the microsphere surface by PECVD at the Korea

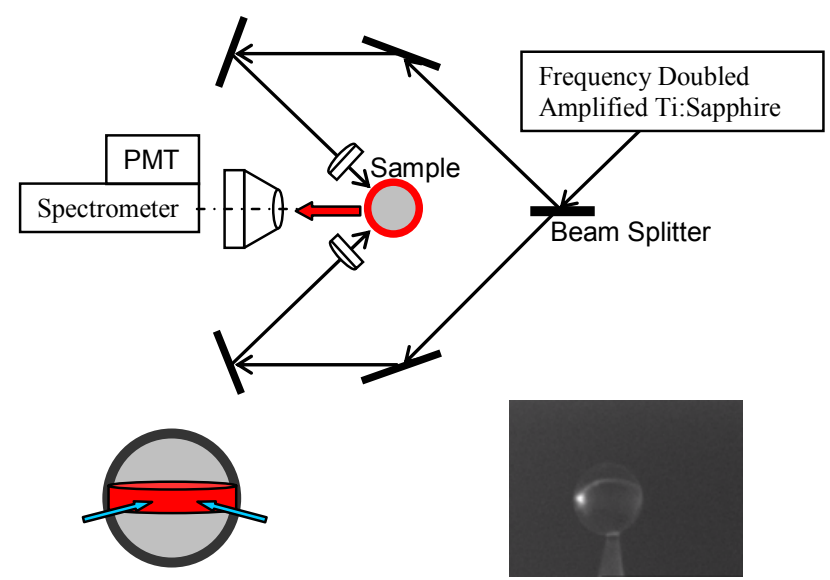

Fig. 1. A sketch of the experimental set up. Two beams crossing at $90^{\circ}$ pump the microsphere. The inset is a PL image.

Advanced Institute of Science and Technology. After deposition the microspheres are first annealed for an hour at $1100{ }^{\circ} \mathrm{C}$ in $\mathrm{Ar}$ gas, then hydrogenated in forming gas (1:9 mixture of $\mathrm{H}_{2}$ and $\mathrm{N}_{2}$ ) at $650{ }^{\circ} \mathrm{C}$ for an hour. The thermal annealing process introduces phase segregation in the $\mathrm{SiO}_{\mathrm{x}}$ layer and transforms it into a silicon nanocrystal embedded silica layer. The sample is then brought to University of Rochester where the following experiments are performed.

An amplified frequency doubled Ti sapphire system with ultrashort pulses (200 fs, $1 \mathrm{kHz}, 405 \mathrm{~nm}$ ) is used to excite the sample. A sketch of the setup is shown in Fig. 1. The incident beam is split into two equal beams. After traveling two symmetric paths, both beams are incident onto the sample surface, from two perpendicular directions. A Two cylindrical lenses are used to focus the beam into a very thin horizontal stripe (about $20 \mu \mathrm{m}$ wide) so that only a narrow ring around the equator of the sphere is under excitation. Using this two-beam cross pumping geometry, a relatively uniform excitation can be achieved along the equator. The photoluminescence (PL) image is recorded by a Hitachi KP200 near infrared camera. The inset of Fig. 1 shows the PL image. Near the equator, a ring shaped PL pattern is clearly noticeable. 
(a)

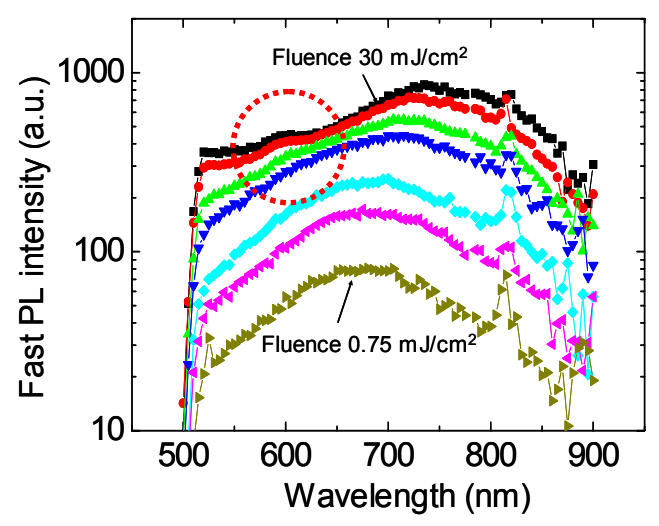

(b)

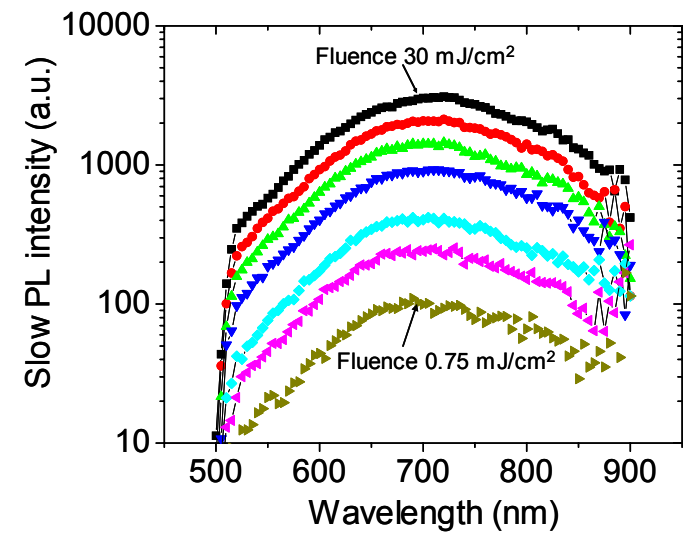

Fig. 2. (a) Fast emission PL spectra. (b) Slow emission PL spectra. The pumping intensities from bottom to top are $0.75,1.9,3.0,7.5$, 12,19 , and $30 \mathrm{~mJ} / \mathrm{cm}^{2}$, respectively. In the fast PL spectra, the sharp peak at $810 \mathrm{~nm}$ results from residual pump laser light that is not frequency doubled.

A spectrometer and a gated photon-counter are used to record the PL spectrum. The excitation energy per pulse is measured to be $3 \mu \mathrm{J}$, focusing into a stripe of $200 \mu \mathrm{m}$ by 20 $\mu \mathrm{m}$. A set of neutral density filters is used to modify the pumping fluence. The maximum fluence applied is 30 $\mathrm{mJ} / \mathrm{cm}^{2}$, and the minimum is $0.75 \mathrm{~mJ} / \mathrm{cm}^{2}$. The PL spectra are taken under different excitation intensities. The fast PL spectra record the signal emitted in the first $50 \mathrm{~ns}$ after excitation and the slow PL spectra record the signal emitted afterwards.

\section{RESULTS AND DISCUSSIONS}

Fig. 2(a) and Fig. 2(b) show the fast and slow emission spectra, respectively. In the fast PL spectra, we notice one especially interesting feature. In the rising edge of the PL spectra, we observe the emergence of a clear peak at 590 $\mathrm{nm}$ when the pumping intensity increases above a certain level. At the same time it is also noticeable that the luminescence in the 550 to $650 \mathrm{~nm}$ range is actively suppressed. This can be seen from the curvature of the spectra, which changes from convex to concave. However, a similar behavior is not observed in the slow emission spectra shown in Fig. 2(b).

If we plot the emission intensity for any wavelength from Fig. 2 vs. the pumping intensity, the trend is sub-linear. However, in our experimental setup we detect the signal from the possible cavity modes as well as any spontaneous emission from the illuminated region. In fact, the detectable intensity from any cavity mode is expected to be weak because we are monitoring the signal that leaks out of the cavity mixed with the large spontaneous emission from the non-cavity area. Considering this, it is necessary to isolate the signal from the background spontaneous emission, and then record the pumping fluence dependence of the isolated signal. A Gaussian fit is applied to the PL spectra to obtain the spontaneous emission signal, which is then subtracted from the total intensity to obtain the isolated signal. Fig. 3 shows the pumping fluence dependence of the isolated intensity around $590 \mathrm{~nm}$. As the pumping intensity increases, we observe the emergence of a narrow (FWHM $40 \mathrm{~nm}$ ) peak around $590 \mathrm{~nm}$. More importantly, as shown in Fig. 4, if we integrate the signal intensity around this $590 \mathrm{~nm}$ peak (from 530 to $680 \mathrm{~nm}$ ), the pump power dependence of the integrated intensity shows a definite threshold with a strong, super-linear (slope 1.9 in log-log scale) increase.

Overall, there are three striking features of this $590 \mathrm{~nm}$ peak. First, the emergence of the peak is accompanied by suppression of the spontaneous emission. Second, the peak width is much narrower than the spontaneous emission FWHM reported in all silicon nanocrystal samples including our microspheres. Last, the peak intensity shows a definite threshold with a super-linear increase afterward. These features match the four commonly recognized signatures of lasing: spectral narrowing, lifetime shortening, a well-defined threshold behavior and a superlinear increase afterward. All this suggests that we have observed stimulated emission in our silicon nanocrystal microspheres. Preliminary control experiments also suggest

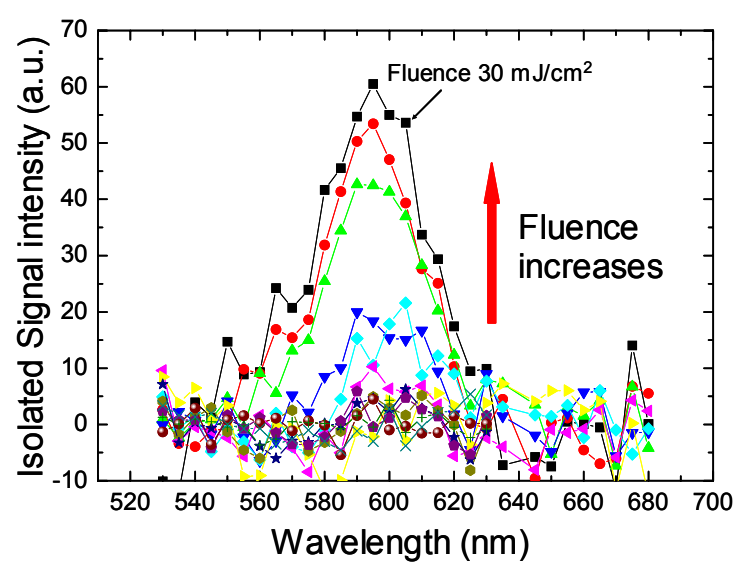

Fig. 3. The signal intensity after background signal subtraction detected immediately after excitation. 


\section{ACKNOWLEDGEMENTS}

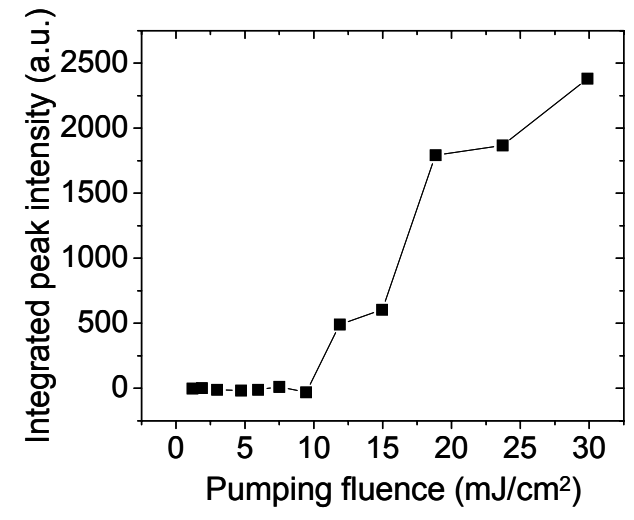

Fig. 4. Integrated peak intensity at $590 \mathrm{~nm}$ vs. pumping fluence.

that the presence of silicon nanocrystals is necessary to observe the stimulated emission signal. The origin of the stimulated emission (e.g., excitonic recombination or defect-related recombination) remains to be determined.

\section{CONCLUSION}

A narrow emission lineshape near $590 \mathrm{~nm}$ is observed in silicon nanocrystal coated microspheres. The features of this emission lineshape are consistent with stimulated emission and lasing.
Financial support from the National Science Foundation and Semiconductor Research Corporation is acknowledged.

\section{REFERENCES}

[1] http://public.itrs.net/

[2] H. Yamada, T. Chu, S. Ishida, and Y. Arakawa, "Optical directional coupler based on Si-wire waveguides", IEEE Photon. Tech. Lett., Vol 17, pp. 585-587, 2005

[3] A. Liu, R. Jones, L. Liao, D. Samara-Rubio, D. Rubin, O. Cohen, R. Nicolaescu, and M. Paniccia, "A high-speed silicon optical modulator based on a metal-oxide-semiconductor capacitor", Nature, Vol. 427, pp.615-618, 2004

[4] B. Yang, J. D. Schaub, S. M. Csutak, D. L.Rogers, J. C. Campbell, "10-Gb/s all-silicon optical receiver," IEEE Photon. Tech. Lett., Vol. 15 , pp. 745-747, 2003.

[5] L. Pavesi, L. Dal Negro, C. Mazzoleni, G. Franzo, and F. Priolo, "Optical gain in silicon nanocrystals", Nature (London), Vol. 408, pp. 440-444, 2000.

[6] L. Dal Negro, M. Cazzanelli, L. Pavesi, S. Ossicini, D. Pacifici, G. Franzo, F. Priolo, and F. Iacona, "Dynamics of stimulated emission in silicon nanocrystals," Appl. Phys. Lett., Vol. 82, pp.4636-4638, 2003.

[7] J. Ruan, P. M. Fauchet, L. Dal Negro, M. Cazzanelli, and L. Pavesi, "Stimulated emission in nanocrystalline silicon superlattices," Appl. Phys. Lett., Vol. 83, pp. 5479-5481, 2003.

[8] S. M. Spillane, T. J. Kippenberg, and K. J. Vahala, "Ultralowthreshold Raman laser using a spherical dielectric microcavity", Nature, Vol 415, pp. 621, 2002.

[9] M. Pelton and Y. Yamamoto, "Ultralow threshold laser using a single quantum dot and a microsphere cavity", Physical Review A, Vol 59, pp. 2418,1998 